\title{
Some Notes on the Controllability in a Steady State for the Q-W Equations With Weak Decay
}

\author{
Shuxian Deng ${ }^{1,2, a}$ and Hongen $\mathrm{Li}^{1, \mathrm{~b}^{*}}$ \\ ${ }^{1}$ Department of Basic Courses, Zhengzhou University of Industrial Technology, Xinzheng, China \\ ${ }^{2}$ College of Science, Henan University of Engineering, Zhengzhou, China \\ adshuxian@163.coml, b857931663@qq.com \\ *The corresponding author
}

Keywords: Steady; Controllability; Weak; State

\begin{abstract}
We present a new method of investigating the so-called quasilinear strongly-damped wave equations in this paper, we concerned with the Controllability in a steady state for the Quasilinear Wave Equations with weak decay, and we use a so-called energy perturbation method to establish weak controllability of solutions in terms of energy norm for a class of nonlinear functions. This method allows us to establish the existence and uniqueness of energy solutions. We also established the existence of finite-dimensional global and exponential attractors for the solution semigroup associated with that equation and their additional regularity. We show the controllability in a steady state with the help of differential inequalities by estimating the relationship between energy inequalities and attenuating property of weak solutions. We determine a small positive number and derive differential inequalities by using a perturbation of energy.
\end{abstract}

\section{Introduction}

We consider the control problem of weak decay to the following so-called quasi-linear wave equation in a smooth bounded domain $\Omega \subset R^{3}$

$$
\begin{aligned}
& u_{t t}-\lambda \Delta u_{t}+u \cdot \nabla u=f(x, t) \\
& \operatorname{div} u=0 \\
& u(x, 0)=u_{0}, \quad u_{t}(x, 0)=u_{1}
\end{aligned}
$$

Where $u_{0}, u_{1}, f$ are given functions, $\Delta u$ is a Laplacian with respect to the variable $x \in \Omega$, $u=u(t, x)$ is an unknown function, $\lambda>0$ is a fixed positive number, $f$ are given external forces, and satisfying the following conditions:

$$
f \in L_{2}, u \in L_{q}\left(R^{+}, L_{p}\right) \text { and } \frac{2}{p}+\frac{n}{q} \leq 1
$$

For some positive $a, p \in[1 / 2,2), C>0$, and $q>0$, we have a weak solution, which fulfills additionally

$$
\begin{aligned}
& d+a|x|^{p} \leq f^{\prime}(x, t) \leq d\left(1+|x|^{q}\right), \forall s \in R^{3} \\
& \text { And } \quad u_{t t}-\operatorname{div} \lambda \nabla u^{2}-\Delta u_{t}=f(x, t)
\end{aligned}
$$

We have several techniques to prove the existence of weak decay solutions with respect to the phase space, and have additional nice properties with energy inequality for almost all times or solutions with weak decay properties for $t \rightarrow \infty$, this has been studied recently by several people, e.g. Y.M. Qin, Ebihara, Xin Liu [1-6] etc.

As a model of quasi-linear wave equation, for $N=1 / 2,1$ and $f=0,1$ equation(1.1)-(1.3)admits a global weak decay solution as large initial data, which was proved by Y.M. Qin, Xin Liu, X.G. Yang, 
Lan Huang etc [3-8]. T.G Wang, Ming Zhang, M.J Wang simplified the above arguments and give the proof of control with exponential decay. From the perspective and background of physics, this represents an implementation of an axial movement of the viscoelastic material, this causes the form of above equations, in the one-dimensional case, their model of longitudinal vibration of a uniform rod with nonlinear stress function $f$. In two, three-dimensional case, they describe the viscoelastic solid of anti-plane shear action. While $n=1$ and $f=0, \mathrm{~S}$.T. Li prove the existence of weak periodic decay strong solution on the periodicity condition, X.K. Su and J.L. Zhang[6-10] proved the controllability of a smooth solution in the method of Cauchy problems in the case of smooth and small data.

Ulteriorly, while $n>\frac{1}{2}$ and $f \neq 0$, M.J. Wang and X.G. Yang gave the proof of global controllability with a smooth solution in the case of small initial data. Make use of combining $L^{p}$-theorem of Sobolev space and semigroup theorem of operators, Nakao [3-4] and A.F and H.B [5-7] devised certain decay rate of the energy of global solutions with large data under a specific condition which is certainly satisfied if the mean curvature of the boundary $\partial \Omega$ is non-positive. For $n>1$ and $f=1$, nonlinear elliptic equation with periodicity conditions was studied [10],

$$
\begin{aligned}
& d^{\prime}(t)+k_{1}\left\|w_{1}(t)-w_{2}(t)\right\|_{p}^{v} d(t) \leq k_{2} d(t)^{p+q-1}+\kappa d(t)^{\mu}\left\|w_{1}(t)\right\|_{2}^{v+1} \\
& w(x, t+\omega)=w(x, t)
\end{aligned}
$$

In the case of $\|w(t)\| \leq k_{0}(1+t)^{-\frac{\lambda}{2}}$, and $\|w(t)\|_{p} \leq \tau(1+t)^{\left(\frac{\tau}{2}+\frac{n}{2}\left(\frac{1}{2}-\frac{1}{p}\right)\right)}$.

Here, $\Omega$ is a bounded domain in $R^{n}$ with a smooth, $\partial \Omega$ is said to be $C^{2}$ class boundary, which satisfies the following uniform hyperbolic assumption:

For some constants $\rho_{0}, M>0, \tau \in H^{4}[0,+\infty)$ satisfies:

$$
\begin{aligned}
& x^{\prime}(t)+\tau_{0}(1+t)^{\left(1+\frac{d}{2}\right)} x(t)^{\left(2+\frac{\gamma}{p}\right)} \leq \sigma y(t)^{\frac{v}{2}}+\tau_{1}(1+t)^{\delta} y(t)^{\gamma} \\
& \tau\left(v^{2}\right)+2 \sigma_{0}\left(v^{2}\right) v+\tau^{\prime \prime}\left(v^{2}\right) \leq M<\infty
\end{aligned}
$$

Thus, we can use a so-called energy perturbation method to establish weak controllability of solutions in terms of energy norm for a class of nonlinear functions.

This method allows us to establish the existence and uniqueness of energy solutions. We also establish the existence of finite-dimensional global and exponential attractors for the solution semigroup associated with that equation and their additional regularity[9-11]. We will show the controllability in a steady state with the help of differential inequalities by estimating the relationship between energy inequalities and attenuating property of weak solutions. And furthermore, we will determine a small positive number and derive differential inequalities by using a perturbation of energy and conclude some results immediately in the next text.

\section{Main Results}

First, we focus on the control of weak decay stability, in order to describe the maneuverability; we define global weak solution with decay for (1.1)-(1.3):

If the initial data satisfies $u_{0}, u_{1} \in H^{2} \cap H_{0}^{4}$, and the function $\omega$ is said to be a weak solution of Problem (1.1)-(1.3), if it satisfies the following conditions:

$$
\begin{aligned}
& \text { (a) } E(t) \equiv \frac{1}{2}\left\{\left\|\omega_{t}(t)\right\|^{2}+\int_{\Omega} \int_{0}^{|\nabla \omega|^{2}} \tau(\xi) d \xi d y+\int_{0}^{t}(f, \omega(t)) d s d y\right\} \\
& \text { (b) }\left.\left(\omega_{t}, \mu\right)\right|_{0} ^{t}+\int_{0}^{t}\left\{\left(\omega_{t}, \mu_{t}\right)-(\operatorname{div}(\tau \nabla \omega), \mu)-\left(\Delta \omega_{t}, \mu\right)-(f, \mu)\right\}=0
\end{aligned}
$$


It is well known that the existence of such a weak solution with decay for all times is assured. Once this is known, one can identify this solution with the global weak one and continue this process to get that

$$
t^{2}\left\|\partial_{t} v\right\|_{H}^{2} \leq \lambda t^{2}\left\|\partial_{t} v\right\|_{L^{\infty}}^{1 / 2}\left\|\partial_{t} v\right\|_{L^{2}}^{3 / 2} \leq \delta t^{3}\left\|\partial_{t} v\right\|_{L^{2}}^{3}+\rho\left\|\partial_{t} \omega\right\|_{L^{\infty}}^{2}
$$

Obviously, we will construct the controllability of the local weak solution with decay for the semigroup generated by weak energy solutions:

Theorem 1. Under the above hypothesis and suppose that $\omega(x . t)$ is a sufficiently regular weak solution of problem (1.1)-(1.3), the following estimates hold

(c)

$$
\left\|\partial_{t}^{2} \omega(\xi)\right\|_{H}^{2} \leq \delta t^{3}\left\|\partial_{t} v\right\|_{L^{2}}^{3}+C^{3} \rho^{2} \delta^{-2}\left\|\partial_{t} \omega\right\|_{L^{\infty}}^{2}+\lambda v(t) e^{\varepsilon(t-\alpha)}
$$

(d) Let the assumptions (1.2) and (1.3) be satisfied with $\theta=2$, and $f(t) \leq \kappa e^{-2 \rho t}$. Then, the local weak solution with attenuation $\omega(t)$ of problem (1.1)-(1.3) which satisfies the additional regularity, and exists constants $\mathrm{M}>0, v>0$ such that

$$
E(t) \leq M e^{-3 v t}
$$

Theorem 2. Let $\omega$ be a weak solution with the decay of (1.1)-(1.3). Denote by $\omega_{0}(t)=e^{-t \Delta}$ the solution of the wave equation and suppose $\left\|\omega_{0}(t)\right\|_{2} \leq \lambda_{0}(1+t)^{-\tau / 2}, \omega_{0} \in H^{2}(\Omega) \cap H_{0}^{4}(\Omega)$, $\omega_{1} \in H_{0}^{4}(\Omega)$ and $f^{\prime}(x, t) \in W_{0}^{2}$ then problem(1.1)-(1.3) hold a unique local weak solution $\omega(t)$ with the following estimate

$$
y^{\prime}(t)+\lambda_{0}(1+t)^{\frac{\kappa}{p}} y(t)^{\left(1+\frac{\varepsilon}{2} \kappa\right)} \leq \lambda_{1} y(t)^{\frac{\gamma}{2} \mu}+\lambda_{2}(1+t)^{-q} \nabla x \omega(t)
$$

Remark. Obviously, from Theorem 1 and Theorem 2, we can easy to establish the control of stability for polynomial decay to (2.3)-(2.5). In comparison to (2.2)-(2.3), we give the strong stability estimates. Add the limit $t \rightarrow \infty$ to the dissipative estimate (2.4) for the approximations $\omega(t)$, and together with Sobolev embedding theorem, we can immediately conclude that the limit weak solution $\omega(t)$ also satisfies:

$$
\|\nabla \omega\|_{2}+\left\|\partial_{t} u\right\|_{L^{2}}^{2} \leq\left\|\partial_{t} u_{1}\right\|_{L^{\infty}}^{2}+\int_{0}^{t}\|\nabla h(x)\|_{L^{2}} d x+\left(\partial_{t} v, v\right)
$$

\section{Proofs of Main Results}

Proof of Theorem 1. Without loss of generality, we consider the initial-boundary value problem for the following nonlinear wave equation:

$$
\begin{aligned}
& u_{t t}-\operatorname{div}\{(a|\nabla u| 2 r+1) \nabla u\}-\Delta u_{t}+\Delta_{x} u=g(x, t) \\
& \left(g(u), \partial_{t} v\right)+\Delta \omega_{n}+m_{0}\left|\nabla \omega_{n}\right|^{q} \leq \varepsilon|v|^{2 q}+C_{\varepsilon}\left\|\partial_{t} v\right\|_{H^{2}}^{2}+m_{0}\left|\nabla v_{n}\right|
\end{aligned}
$$

Analogously, for some positive $\varepsilon, v, \rho$, together with the Hölder inequality and the interpolation

$$
g^{\prime}(t)+C g(t)^{1+\frac{v}{p}} \leq C(1+t)^{-\rho}(1+t)^{\frac{v d}{2 n}} g(t)^{v} \leq \varepsilon\left\|\partial_{t} v\right\|_{L^{2}}^{2} C_{\varepsilon}\left\|\partial_{t} v\right\|_{H^{2}}^{p+1}
$$

Similarly as above, we derive the differential inequality as long as the local weak solution $u(t)$ exists, inserting the above estimates, and using the energy estimate for estimating the energy norms, one can get

$$
\left\|\partial_{t} u\right\|_{L^{2}}^{n p-\gamma}+\frac{\gamma}{2}\left\|\nabla_{x} u\right\|_{L^{2}}^{\frac{1}{v}+\frac{d}{2}}-a\|\nabla u\|_{L^{2 r}}^{2 r} \leq-C_{2} E(t)+C_{3}\|g\|^{2}-\frac{C_{4}}{2} \Phi+\frac{\varepsilon^{2}}{4} M\|u\|^{2}
$$

On the other hand, by Young's inequality, choosing $\varepsilon>0$ small enough and $E(t)$ is bounded, we finally deduce the following 


$$
\begin{aligned}
& G_{0}^{\prime}(t)+C_{3} G(t) \leq C_{4} M_{1}(1+t)^{-\rho_{1}} \\
& g^{\prime}(t)+C_{4}(1+t)^{\frac{\lambda \rho}{2}} g(t)^{\frac{1+\lambda}{p}}<C_{5}(1+t)^{-\frac{1}{1+\gamma}} g(t)^{\beta}
\end{aligned}
$$

Using now estimate (3.2)-(3.4) together with the interpolation inequality, we infer from (3.5)-(3.6) that

$$
g^{\prime}(t)+C_{0} \mathrm{~g}(t)^{p+2-v}+\left\|\xi_{u}(y)\right\|_{L^{2}}^{p+2} \leq C_{6} e^{K(L-s)}\left\|\xi_{u}(x)\right\|_{L 2}^{p+2-n}+C_{7} g(t)^{\frac{n}{p(p+2)}}
$$

Now fix $L, S$.Then, Taking advantage of the smoothing property together with the obviously bounded $E(t)$

$$
E(t)+C_{7} \Phi(0)+G_{0}(t) \leq 2 \Phi(t) \leq 2 g(t)+2 K G(0)(1+t)^{-K}+C_{8} M_{3} e^{-\lambda t}
$$

Multiplying the both sides of (3.7) by $e^{C t}$ and integrate from 0 to $t$, we derive

$$
C_{6} \Phi(s)+\int_{B\left(x_{0}, r\right)} \tau^{2} g\left(u_{k}\right) p(x)\left|\nabla u_{k}\right|^{q} d x \leq \int_{B\left(x_{0}, r\right)} \delta^{2} f\left(u_{k}\right) p(x) \Delta u_{k} d x
$$

We finally obtain

$$
E(u(t)) \leq 2 G(u(t)) \leq 2 C_{\varepsilon} e^{-C_{\varepsilon} t} \leq C_{\sigma} E\left(e^{-\lambda_{\sigma} t}\right)
$$

Hence, the theorem is completed.

Proof of Theorem 2. in fact, for $n=3, \omega \in L_{1} \cap L_{p}$, and $\left\|v(t)-v_{0}(t)\right\| \leq \sigma(1+t)^{\frac{\gamma}{3}\left(1-\frac{1}{q}\right)}$, note that the initial data is dense, hence $\left\|v_{0}(t)\right\|$ attenuates exponentially fast, Indeed, using $\omega|\omega|^{\frac{2 p}{n(p-2)}}$ and $\partial_{t} \omega$ multiply the equation (1.7) and integrate by parts over $x \in \Omega$. One gets

$$
\delta^{\prime}(t)+\lambda_{1}(1+t)^{\kappa \frac{d}{2}} \delta(t)^{\frac{1}{q}} \leq \varepsilon(1+t)^{-(p-r)} y(t)^{\lambda+\frac{\kappa}{p}}+\lambda_{2} \delta(t)^{\frac{n}{2}\left(\frac{1}{r}-\frac{1}{2}\right)}
$$

Where $\varepsilon$ is a small positive number which will be fixed, Then we arrive at

$$
\frac{1}{2}(p-n) \delta^{\prime}(t)+\int_{\Omega}\left|\nabla \omega^{2}\right|^{p} d x \leq \sigma_{\varepsilon} \int_{\Omega}\left(\left|v_{0}\right|^{4}+|\nabla \omega|^{p-2}\right) d x+\frac{1}{2} v(G(v), v)
$$

And notice that

$$
\begin{aligned}
& E(u(t))=\frac{1}{2} \alpha\left\{\left\|\nabla \omega_{1}(t)\right\|^{q}+\int_{\Omega} \int_{0}^{\left|\nabla u_{1}\right|^{2}} \sigma(\xi) \omega(t) d \xi d x\right\} \\
& E\left(\xi_{u}(t)\right)=\frac{p-2}{p}\left\|\partial_{t} \omega\right\|_{L^{2}}^{p}+-\left(G(\omega), \frac{1}{q-1}\right)-\frac{p n+4}{p+4-n}\|\nabla \omega\|_{L^{2}}^{2}
\end{aligned}
$$

Let $\lambda, \alpha$ be small enough, one gets

$$
\frac{d}{d t} E\left(\xi_{u}\right)+\rho\left\|\nabla_{x} u\right\|_{H_{0}^{2}}^{p}-\alpha\left\|\partial_{t} u\right\|_{H_{0}^{2}}^{2}=\mu\left(\varphi^{\prime}\left(\nabla_{x} u\right), \nabla_{x} u\right)+\beta(g(u), \omega)
$$

And

$$
\int|u|^{\frac{p n}{n-2}} d x \leq\|\omega\|_{H_{0}^{2}}^{\frac{p}{2}-1}+\sigma\left\|\nabla_{x} u\right\|_{L^{p}}^{2} \leq \frac{C_{\varepsilon}}{\beta} \zeta(\omega)+\lambda_{\varepsilon}\|\omega\|_{L^{p}}^{\gamma}+\delta E\left\|\xi_{u}(t)\right\|
$$

Where the constant $C_{\varepsilon}, \lambda_{\varepsilon}$ depend only on the $\varepsilon$.

Hence by the standard Galerkin method, interpolation inequality, Young's and Sobolev's inequality, this section can be estimated by 


$$
\alpha\|u\|_{L^{q}}^{n+1}\left(\varphi^{\prime}\left(\xi_{2}\right)+\|u\|_{L^{2}}^{\frac{1}{2}-\frac{1}{p}}\right) \leq \alpha\left\|u_{0}\right\|_{L^{2 p}}^{p+2}\left\|v_{0}\right\|_{L^{p}}^{p-1}-\varphi^{\prime}\left(\xi_{1}\right)+C_{\varepsilon}\|u\|_{L^{p}}^{\lambda p}
$$

By the properties of heat kernel, we deduce that

$$
\begin{aligned}
& \partial_{t}\left[\frac{p n-2 n+2 p \alpha}{p+4-n}\left(\partial_{t} v, v\right)\right]+\tau_{0}\left(\left|\nabla_{x} \omega_{1}\right|+\left|\nabla_{x} \omega_{2}\right|\right)^{\frac{p n}{n-2}} \leq C_{1} \varphi(t)^{\frac{1}{p}}+C_{2}(1+t)^{-\mu \tau}|v|^{p+2} \\
& \left.\rho\left\|\partial_{t} u\right\|_{L^{2 p}}^{p+\mu}+C\|u\|_{L^{2}}^{p} \leq \frac{1}{2} \kappa \varphi^{\prime}\left(\nabla v_{1}\right) \leq \mathrm{C}(1+t)^{-p v}+\mathrm{C}_{\varepsilon}(1+t)^{-\frac{n}{2}\left(\frac{1}{q}-\gamma\right.}\right)
\end{aligned}
$$

Noting the uniqueness of energy solution and the Lipschitz continuity in a weak space, there holds (2.6) and Th. 2 is completed.

\section{Acknowledgements}

This work is supported by the Ph.D. Foundation of Henan University of Engineering (No. D2010012).

\section{References}

[1] Y.M. Qin. Global existence of a classical solution to a nonlinear wave equation, Acta Math. Sci.17 (2013), p. 121-128.

[2] Yu-ming Qin, Xin Liu, Shu-xian Deng. Decay Rate of Quasilinear Wave Equation with Viscosity. Acta Math App Sinica, 17 (2010), 147-152.

[3] Nakao, M. Energy decay for the quasilinear wave equation with viscosity. Math. Z., (2015), 219: p. 289-299

[4] Nakao, M. On strong solutions of some quasilinear wave equation with viscosity. Advances in Mathematical Sci. Appl., 6: 267-278 (2013).

[5] A. Friedman, J. Necas, Systems of nonlinear wave equations with nonlinear viscosity, Pacific J. Math. 135 (2008), p. 29-55.

[6] H. Beirao da Veiga, Existence and asymptotic behavior for strong solutions of the N-S equations. Indiana Univ. Math. J., 36(1987), p. 149-166.

[7] Hoff D., Global existence for ID, compressible, isentropic Navier-Stokes equations with large initial data, Trans. Amer. Math. Sot. 303 (2011), p.169-181.

[8] Matsumura A., Large-time behavior of one-dimensional motions of compressible viscous gas, in Recent developments in theoretical fluid mechanics (Paseky, 2012), Vol. 291, Pitman Res. Notes Math. Ser., 1992, pp. 103-128.

[9] Collela, P., Majda, A., Roytburd, V.: Theoretical and numerical structure for reacting shock waves. SIAM J. Sci. Stat. Comput. 7(2016), p.1059-1080

[10] Chen, G.-Q., Hoff, D., Trivisa, K.: Global solutions of the compressible NavierStokes equations with large discontinuous initial data. Comm. Partial Diff. Eqs. 25(2010), p. 2233-2257.

[11]W. Strunk Jr., E.B. White, The Elements of Style, third ed., Macmillan, New York, 2015, pp. 371-395. 